$10-2000$

\title{
The Influence of Orthotic Devices and Vastus Medialis Strength and Timing on Patellofemoral Loads During Running
}

\author{
R. R. Neptune \\ University of Calgary, neptune@roses.stanford.ed
}

I. C. Wright

University of Calgary

Antonie J. van den Bogert

Cleveland State University, a.vandenbogert@csuohio.edu

Follow this and additional works at: https://engagedscholarship.csuohio.edu/enme_facpub

Part of the Biomechanical Engineering Commons

How does access to this work benefit you? Let us know!

\section{Publisher's Statement}

NOTICE: this is the author's version of a work that was accepted for publication in Clinical Biomechanics. Changes resulting from the publishing process, such as peer review, editing, corrections, structural formatting, and other quality control mechanisms may not be reflected in this document. Changes may have been made to this work since it was submitted for publication. A definitive version was subsequently published in Clinical Biomechanics, 15, 8, (10-01-2000); 10.1016/S0268-0033(00)00028-0

\section{Original Citation}

Neptune, R. R., Wright, I. C., and van den Bogert, A. J., 2000, "The Influence of Orthotic Devices and Vastus Medialis Strength and Timing on Patellofemoral Loads during Running," Clinical Biomechanics, 15(8) pp. 611-618.

This Article is brought to you for free and open access by the Mechanical Engineering Department at EngagedScholarship@CSU. It has been accepted for inclusion in Mechanical Engineering Faculty Publications by an authorized administrator of EngagedScholarship@CSU. For more information, please contact library.es@csuohio.edu. 


\title{
The influence of orthotic devices and vastus medialis strength and timing on patellofemoral loads during running
}

\author{
R.R. Neptune ${ }^{\text {a,* }}$, I.C. Wright ${ }^{\text {a }}$, A.J. van den Bogert ${ }^{\text {b }}$ \\ ${ }^{a}$ Human Performance Laboratory, The University of Calgary, Calgary, AB, Canada T2N $1 N 4$ \\ ${ }^{\mathrm{b}}$ Department of Biomedical Engineering, The Cleveland Clinic Foundation, Cleveland, OH 44195, USA
}

\section{Introduction}

Running is a popular form of recreation and fitness and is central to many sports. However, running is also associated with a high injury rate for both younger

\footnotetext{
${ }^{*}$ Corresponding author. Present address: Rehabilitation R\&D Center (153), VA Palo Alto Health Care System, 3801 Miranda Avenue, Palo Alto, CA 94304, USA.

E-mail address: neptune@roses.stanford.edu (R.R. Neptune).
}

$(32 \%)$ and older $(41 \%)$ age populations [1]. Depending on the study cited, the overall yearly incidence rate for running injuries is between $37 \%$ and $56 \%$ [2] with $42 \%$ of these injuries related to the knee and $26 \%$ of knee injuries associated with patellofemoral pain (PFP) [3]. PFP includes all disorders associated with discomfort on the anterior side of the knee joint. Two of the more common, conservative PFP treatments are exercise programs and the prescription of orthoses [4]. Although conservative treatments have been reported to be 
successful, the effectiveness is often based on subjective or empirical data [5]. Therefore, there is considerable disagreement within the scientific community on the mechanisms causing PFP and consequently, it is not clear which treatment is the most effective for specific patients [5-7]. Further, running mechanics and musculoskeletal properties vary among individuals and may play an important role in the effectiveness of individual treatments.

Studies have suggested that differences in relative muscle forces exerted on the patella between the vastus medialis (VM) and vastus lateralis (VL) contribute to lateral patellar tracking and malalignment, which have been linked to PFP [4-7]. The lateral tracking and malalignment occurs when the lateral pull of VL is not adequately balanced by pull of the vastus medialis longus (VML) and vastus medialis oblique (VMO). This imbalance can lead to altered patellofemoral contact forces and pressures that may lead to PFP. The VMO is deemed the primary medial patellar stabilizer due to the anatomical differences between the VML and VMO [8]. Therefore, much effort has been directed towards identifying techniques to selectively strengthen the VMO to improve the force balance on the patella $[4,5,7]$, although the effectiveness and feasibility of selective VMO strengthening have been widely questioned $[6,7,9,10]$. Much of the controversy regarding the effectiveness of VMO strengthening is caused by the difficulty of isolating the treatment effect in patients. Electrical stimulation may be the only way to strengthen the VMO selectively [11], but should not be recommended unless its effect on patellofemoral joint mechanics is shown to be significant. Therefore, a theoretical analysis is needed to quantify the effect of VMO strengthening on patellofemoral joint loads to justify further studies seeking to identify techniques to selectively strengthen the VMO.

From a neuromechanical perspective, selective VMO activation can also reduce the lateral dominance of $\mathrm{VL}$ by either increasing the intensity of VMO relative to VL, or by initiating VMO activity prior to VL [6]. In patients with PFP, it is speculated that VL is activated earlier than VMO [6,12] and at a higher relative intensity [13]. But studies examining this dynamic imbalance theory have provided conflicting results [7,12-16], and the functional significance of the identified timing differences, on the order of $5 \mathrm{~ms}$, has been questioned [6,7]. Methodological differences between studies and the inherent difficulty identifying muscle excitation onset and offset make reconciling differences difficult, but these studies suggest a theoretical investigation into the influence of vasti timing and intensity is warranted.

Another conservative treatment for PFP has been the prescription of foot orthoses [4]. Eng and Pierrynowski [17] found that foot orthoses combined with an exercise program can be an effective method to reduce PFP in young females. Bahlsen [18] examined the relationship between excessive foot pronation and PFP and found a significant association. The mechanism behind the pain is speculated to be a kinematic coupling between excessive foot pronation and prolonged tibial rotation [1820]. Tiberio [20] used a theoretical model to examine the relationship between excessive pronation and lateral patellofemoral joint compression. Tiberio [20] suggested that during gait, the prolonged tibial rotation prevents the knee from extending freely. To compensate for this, the femur rotates internally relative to the tibia causing changes to the patella contact force and pressure patterns. This biomechanical explanation was termed compensatory internal rotation of the femur, although other studies have provided alternative explanations of the relationship between excessive foot pronation and PFP [5]. These studies suggest that further research is needed to understand the biomechanical relationship between excessive pronation and PFP before the effectiveness of foot orthoses verses exercise programs can be evaluated.

Running is a highly dynamic and complex movement that makes it difficult to predict the effect of these treatments on musculoskeletal loading during movement. Changes in the movement caused by these mechanical treatments (e.g., orthoses, [21]) result in changes in the muscle kinematics and therefore the muscle forces through the intrinsic muscle force, length and velocity relationships. The dynamic interaction within the musculoskeletal system makes such changes difficult to predict and interpret and are often counterintuitive [22]. Thus, the mechanisms behind the changes in patellofemoral joint loading are difficult to identify.

Forward dynamic simulations of running have been developed that allow for the precise identification of muscle forces and joint loading and have contributed to the understanding of mechanisms involved in loading the leg during running (e.g., [23-25]). These models were, however, limited to the initial impact phase $(0$ $50 \mathrm{~ms}$ ). Since the etiology and mechanics of PFP and the effectiveness of conservative treatments are not well understood, a theoretical model and simulation approach may contribute to our understanding and provide the information necessary to design effective rehabilitation protocols. Therefore, the goal of this study was to use a three-dimensional forward dynamic simulation of running to investigate the effectiveness of selective VMO strength and activation and the prescription of orthoses on patellofemoral loads.

\section{Methods}

\subsection{Musculoskeletal model}

A forward dynamic musculoskeletal model was used to produce simulations of heel-toe running. The model 
was previously described in detail [26] and will be described briefly here. The musculoskeletal model was developed using DADS 8.5 software (CADSI, Coralville, IA, USA) and consisted of rigid segments representing the foot, talus, shank, patella, thigh of the support leg, pelvis, and a rest-of-body segment. The model was dimensioned to represent a male subject with a height of $180 \mathrm{~cm}$ and a mass of $75 \mathrm{~kg}$. Musculoskeletal geometry was based on the work of Delp [27] and segment masses and inertial properties were determined using regression equations $[28,29]$.

The joint models were based on existing literature [27] and further developed to allow three-dimensional movements at the knee. The hip joint was modeled as a spherical joint while rotations about the subtalar and talocrural joints were allowed at the ankle [30]. The tibiofemoral joint was modeled with three degrees of freedom with a moving center of rotation for flexion/ extension and appropriate passive stiffness for ab-adduction and internal/external rotation. The patella was constrained to move along a prescribed trajectory relative to the femur [27]. The rotation and anterior/posterior translation were functions of the superior/inferior translation relative to the femur. The patella was attached to the tibia by an inextensible patellar tendon. The foot consisted of three segments: rear-foot, mid-foot and toes. Flexion/extension and internal/external rotations were allowed between the rear-mid and mid-foot, and flexion/extension was allowed between the mid-foot and toes. Passive stiffness torques were applied at these joints so that realistic displacements were achieved during the mid-stance. Only translation, controlled by a passive spring and damper, was allowed between the pelvis and the rest-of-body segment. The model had a total of 20 degrees of freedom.

Fourteen functionally independent muscle groups were used to drive the model (Fig. 1). The vasti muscle group consisted of four individual muscles all receiving the same muscle excitation; the VL, VML, VMO and vastus intermedius with geometry based on Javadpour et al. [31]. The force-length-velocity characteristics of the muscles were represented by a Hill-based lumped parameter model (e.g., [32]). The complete set of musculoskeletal model parameters can be found in Neptune et al. [26].

\subsection{Ground contact model}

The contact between the foot and the ground was modeled by 66 discrete independent visco-elastic elements, each attached to one of the three foot segments in locations that describe the three-dimension exterior surface of a shoe when the foot joints are in a neutral position (Fig. 2). Each element permitted deformation perpendicular to the floor and represented the mechanical properties of the shoe sole and underlying soft tis-

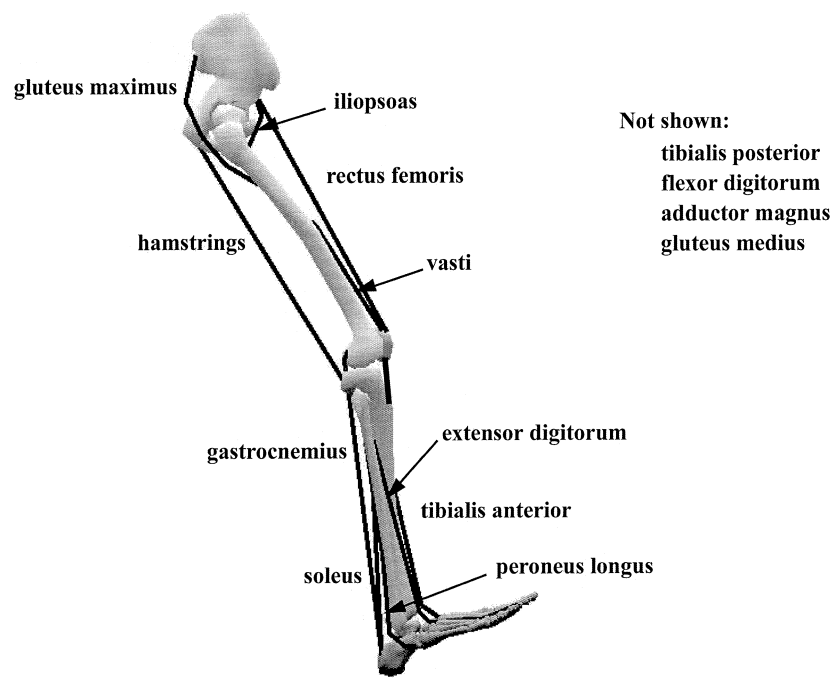

Fig. 1. Musculoskeletal simulation model. The muscles included in the model were the gluteus maximus (GMAX), iliopsoas (PSOAS), adductor magnus (ADDMAG), gluteus medius (GMED), hamstrings (HAMS), rectus femoris (RF), vasti lateralis, intermedius, medialis longus and medialis obliques (VAS), gastrocnemius (GAS), peroneus longus (PER), soleus (SOL), tibialis posterior (TIBPOST), tibialis anterior (TA), extensor digitorum longus (EXTDIG) and flexor digitorum longus (FLEXDIG). The four vasti muscles all received the same excitation signal.

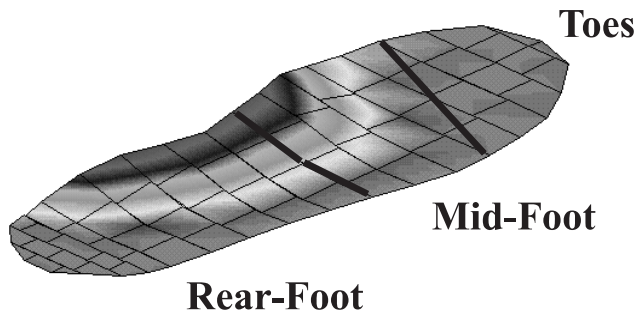

Fig. 2. Array of ground contact elements on the rear-foot, mid-foot and toes representing a soft running shoe. The solid lines crossing the foot segment indicate the intersections between the rear-foot, mid-foot and toes. The point of contact is the center of each rectangular area.

sue. The anterior-posterior, medial-lateral, and vertical force calculations as well as the determination of shoe specific parameters were presented in detail [26].

\subsection{Subject specific simulations}

Subject specific simulations were produced by searching for the muscle excitation patterns that minimized the difference between simulated and experimentally measured segment orientations and external ground reaction force profiles (Eq. (1)) for each subject $(n=9)$ using a simulated annealing optimization algorithm [33]. The objective function to be minimized was defined as

$J(p)=\sum_{j=1}^{m} \sum_{i=1}^{n} \frac{\left(Y_{i j}-\hat{Y}_{i j}\right)^{2}}{S D_{j}^{2}}$, 
where is the measurement of variable $j$ at time step $i$, the simulation data corresponding to , 2 the average inter-trial variability of variable $j$, and $=\left(\begin{array}{lll}1 & \cdots & 42\end{array}\right)$ are the parameters describing the neural excitation patterns.

A similar form of the objective function (Eq. (1)) was used in a previous pedaling simulation study [34] and effectively produced simulations that reproduced experimental data. The specific quantities evaluated in Eq. (1) were the four Euler parameters describing the global attitude matrix of the rear-foot, shank and thigh, and the three $\left(\begin{array}{lll}x & y & z\end{array}\right)$ components of the ground reaction force, resulting in a total of $m=15$ variables included in the objective function (Eq. (1)). The optimization was terminated when the cost function did not decrease the cost function by $1 \%$ within 500 function calls. This optimization procedure was used to obtain nine different simulations of running stance, based on movement data from nine different subjects.

\subsection{Movement data}

Experimental data were collected from a group of nine healthy male subjects (height $=177.0 \mathrm{~cm}$ (S.D., 10.4); weight $=73.3 \mathrm{~kg}$ (S.D., 12.0), age $=22.2$ years (S.D., 2.1)) during heel-toe running to provide initial conditions for the simulation (positions and velocities of the body segments at heel strike) and tracking data ( $Y$ ) for the optimization algorithm. The subjects volunteered to participate in the study and informed consent was obtained before the data collection.

The subjects performed ten trials of heel-toe running at $4.0 \mathrm{~m} / \mathrm{s}$, while kinematic and ground reaction force data were collected. A high speed video system (Motion Analysis Corp., Santa Rosa, CA, USA) was used to record the three-dimensional motion of the body segments at $240 \mathrm{~Hz}$. Three retro-reflective markers were attached to each subject's right shoe (lateral head of the fifth metatarsal, posterior heel, superior lateral aspect of the navicular), shank (head of fibula, anterior mid-shaft of tibia, and distal fibula just proximal to the lateral maleolus), thigh (greater trochanter, anterior mid-thigh, lateral femoral epicondyle) and pelvis (left and right anterior superior illiac spines, and right posterior superior illiac spine). The marker data were used to reconstruct the position and orientation of each body segment [35]. From the limb segment orientations, hip and knee angles were determined using the joint-coordinate system of Grood and Suntay [36] and the subtalar and talocrural joint angles were determined using the jointcoordinate system of Inman [30].

Ground reaction force data were collected simultaneously with the kinematic data at $2400 \mathrm{~Hz}$ using a force platform (Kistler Instumente AG, Winterthur, Switzerland). The time of touch-down was determined when the vertical ground reaction force first exceeded $20 \mathrm{~N}$ and toe-off was indicated when the vertical ground reaction force fell below $20 \mathrm{~N}$. All trials were normalized to the duration from heel strike to toe-off, and the forces and joint angles were sampled at 100ths of this stance duration. The forces were then normalized to body weight. Neither the kinematic nor the ground reaction force data were filtered, but only averaged across trials.

\subsection{Simulation experiments}

Experiments were performed with the nine subject specific simulations to examine the influence of vastus medialis oblique strength (VMOS), vastus medialis oblique excitation timing (VMOT) and orthoses (ORTH) on lateral patella-femoral loads. VMOS was increased by $10 \%$ from the normal strength and VMOT was both delayed and advanced $5 \mathrm{~ms}$ relative to the other vasti muscles. To examine the influence of ORTH, a medial arch orthosis obtained from a sports rehabilitation clinic was modeled in the shoe by increasing the stiffness of the individual shoe elements in the medial arch area in the pattern of the orthosis (Fig. 2). Once the stiffness contour replicating the orthosis was generated, the stiffness values were uniformly scaled until changes in the ankle joint kinematics were similar to those experimentally measured [21]. The lateral patella-femoral joint load was defined as the medio-lateral component of the joint constraint force vector that keeps the patella in the femoral groove and extracted from the simulation data using the DADS 8.5 post-processor.

To assess the effect of these treatments on lateral patellofemoral loads, a two-way mixed factor analysis of variance was performed on the peak and average lateral joint contact forces $(P<0.05)$. When significant treatment effects were detected, a Bonferroni pairwise comparison was performed to identify which treatments were significantly different from the normal condition $(P<0.05)$.

\section{Results}

The optimization was able to find the muscle controls (excitation onset, offset and magnitude) to reproduce the salient features of the experimentally collected data for the nine subjects. Typical simulation results have been previously reported [26]. The major joint angles and ground reaction forces were almost always within 2 S.D. of the subject's data, and the muscle stimulation patterns compared well with the collected EMG data.

Typical medio-lateral patellofemoral loading patterns are shown in Fig. 3. In this subject, there was an impact peak on the lateral side, followed by a phase of medial loading and a second phase of lateral loading during the active push-off. The impact loading was on the lateral side of the joint for all subjects, except for two subjects 


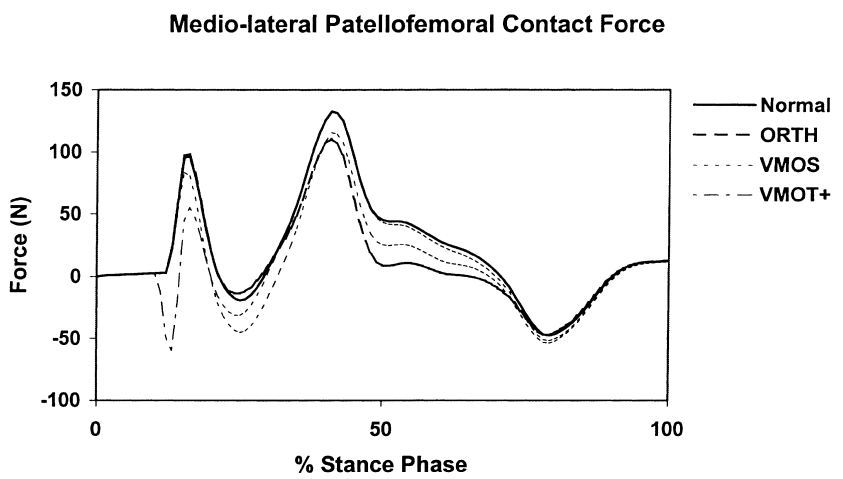

Fig. 3. Typical simulated mediolateral loading patterns for the patellofemoral joint during the stance phase of running. The following treatments were applied: ORTH (orthosis), VMOS (strengthening of the vastus medialis oblique muscle), and VMOT+ (time advance of activation of the vastus medialis oblique muscle).
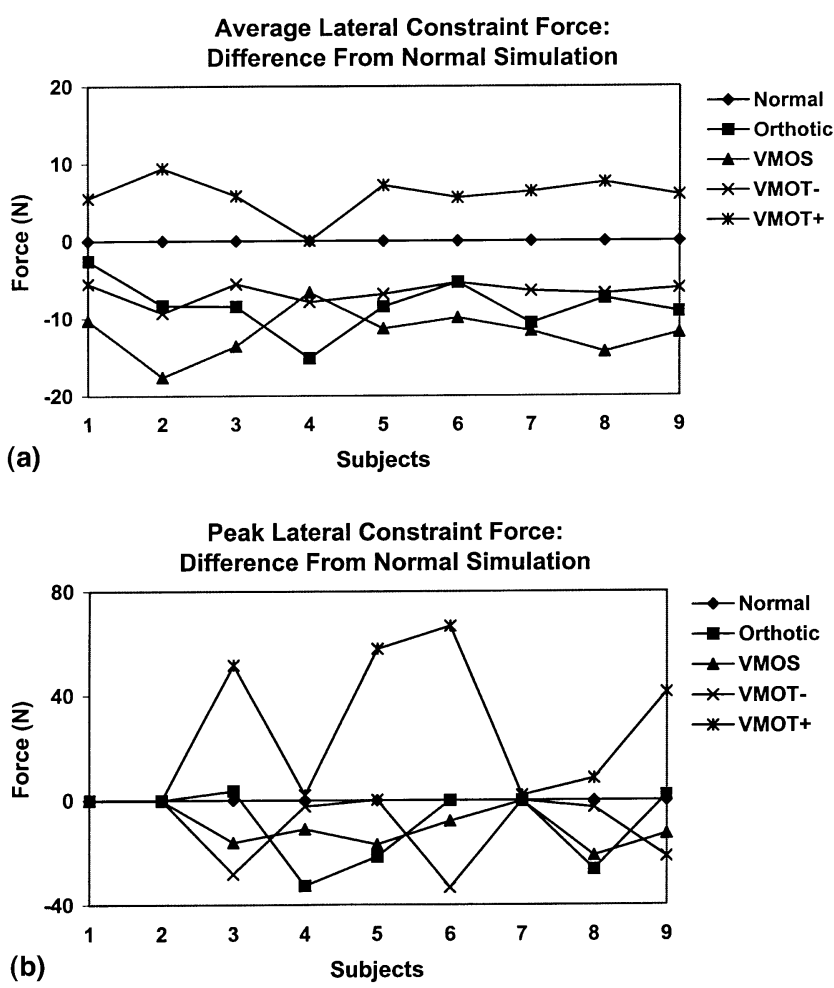

Fig. 4. Average and peak lateral loads in the patellofemoral joint. Values represent the difference in the joint load relative to the normal simulation. Positive and negative values indicate an increase and decrease in the constraint force, respectively.

who had no impact peak. A lateral active peak occurred in four of the nine subjects, and in three of those this peak was higher than the impact peak (in the control condition). The impact peak was never affected by the orthosis, and the active peak was not or only slightly affected by VMO activation timing.

The effect of the four treatments on the peak and average lateral patellofemoral load for the group is presented in Fig. 4. The treatments ORTH, VMOS, and
Table 1

Group average ( $N=9$, S.D. in parentheses) of the peak and average lateral constraint force for the normal condition and the four treatments: VMOS, VMOT+, VMOT- and ORTH ${ }^{\mathrm{a}}$

\begin{tabular}{lll}
\hline Condition & $\begin{array}{l}\text { Peak lateral contact } \\
\text { force }(N)\end{array}$ & $\begin{array}{l}\text { Average lateral } \\
\text { contact force }(N)\end{array}$ \\
\hline Normal & $230(206)$ & $-5(236)$ \\
ORTH & $221(198)$ & $-14(235)^{*}$ \\
VMOS & $220(200)^{*}$ & $-17(237)^{*}$ \\
VMOT + & $220(204)$ & $-12(236)^{*}$ \\
VMOT- & $255(208)^{*}$ & $1(234)^{*}$ \\
\hline
\end{tabular}

${ }^{a}$ Note: a negative value for average lateral contact force indicates that contact occurred more on the medial than on the lateral side, when averaged over the stance phase.

${ }^{*}$ Significantly different from the normal condition $(P<0.05)$.

vastus medialis oblique excitation timing advance $(\mathrm{VMOT}+)$ produced either a reduction in lateral joint loading or no effect at all. The VMO time delay produced an increase in lateral joint loading, which was about twice as large as the decrease produced by the VMO time advance. The statistical analysis revealed that VMOS was the only treatment to significantly reduce the peak lateral constraint force (Table 1). Although ORTH and VMOT+ decreased the constraint force, the effect was not statistically significant. Vastus medialis oblique excitation timing delay (VMOT-) significantly increased the peak force across all subjects. For the average lateral constraint force, all treatments had a significant effect with ORTH, VMOS and VMOT+ decreasing the lateral force while VMOT- increased the load (Table 1). Table 1 also illustrates the large variation in joint loading patterns between the modeled subjects.

\section{Discussion}

The goal of this work was to examine the influence of two commonly prescribed treatments (vastus medialis strengthening and orthoses) for PFP and the functional significance of timing deficits between VMO and VL on lateral patellofemoral joint forces. The forward dynamic simulation model used in the present study had been shown to closely replicate experimentally collected kinetic, kinematic and EMG data during the same running conditions as the present study [26]. The close agreement between the subject and model kinematic, ground reaction force and muscle stimulation data indicated that the model's performance was indistinguishable from the corresponding human subject. In addition, the model's behavior is based on a combination of well-established knowledge: musculoskeletal anatomy, Newtonian mechanics, and Hill's three-component muscle model. These considerations provided 
confidence in the model for experiments examining the effectiveness of common treatments for PFPs that cannot be performed on human subjects. The simulation approach also allowed for the explicit examination of the orthosis effect without the confounding effect of muscle coordination adaptation.

A potential limitation of our simulation approach is that the musculoskeletal model was not customized to the anatomy of individual subjects. It is well known that joint anatomy may predispose individuals to overuse injuries. However, having nine models with identical anatomy but different running mechanics allowed us to unequivocally attribute different responses to treatments to differences in running mechanics rather than anatomy. Such insight is clinically important since running mechanics is more easily altered than anatomy. A potential limitation of this approach is that all joint contact force results were reported using the same femoral joint coordinate system. Specifically, the lateral force was taken as the force perpendicular to the femoral sagittal plane, which contained the patellofemoral groove. This plane may have had a different orientation in different subjects. Therefore, we cannot have confidence in the absolute magnitudes of the lateral constraint force. However, the objective of this investigation was to examine the effectiveness of the treatments and identify mechanisms associated with the reduction in joint loading. To this end, comparisons were performed with the normal simulation and the emphasis was placed on the differences (Fig. 4).

The results showed that VMOS had the greatest effect in reducing the peak lateral constraint force and all treatments were effective in decreasing the average lateral constraint force except VMOT+ (Table 1). Although not presented in the results, the treatments were also applied in combination which showed that the joint loading response was linear (i.e., the reduction or increase in joint loading was additive). Therefore, there did not appear to be any non-linear coupling associated with the combinations of treatments.

Increasing VMO strength was shown to be the most effective treatment for reducing both the peak and average lateral constraint forces. A post-hoc sensitivity analysis was performed on the degree of strengthening from $-50 \%$ to $+50 \%$ that showed the reduction in the lateral constraint force decreased linearly with increased strength (Fig. 5). These results would seem to support the treatment as a potentially effective method in reducing lateral patellofemoral loading. While clinical studies have shown that methods including selective electrical stimulation and biofeedback can produce appreciable results [11,37-39], selective VMO strengthening through isometric and isokinetic exercises has been widely questioned $[6,7,9,10]$. Nevertheless, the theoretical results of the present study suggest future research might be usefully directed towards
Peak Lateral Contact Force

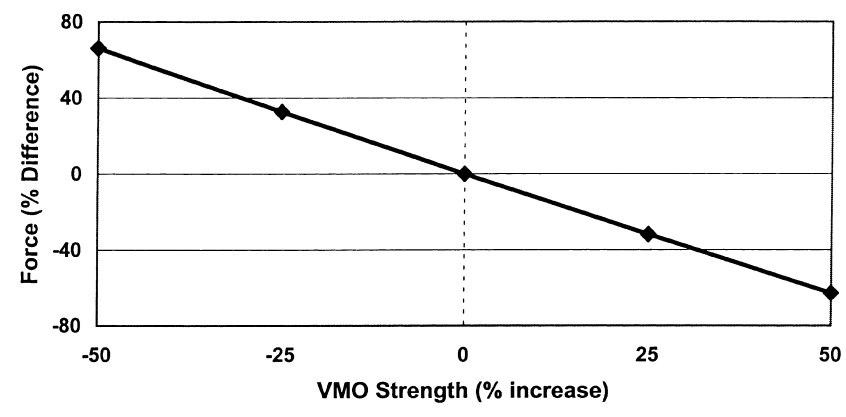

Fig. 5. Sensitivity of the peak lateral patellofemoral load to increases in VMOS for a typical subject. VMOS was systematically increased from $-50 \%$ to $+50 \%$ relative to the normal muscle strength that showed a linear association between VMOS and the magnitude of the lateral joint load.

identifying effective techniques to selectively strengthen the VMO.

The relative intensity and timing of VMO activity relative to VL activity has also been suggested to play an important role in reducing the lateral loads in the knee joint [6]. Although we did not explicitly assess the effect of increased VMO intensity, this was indirectly accounted for in the VMOS experiment. Since muscle force generation is scaled by the magnitude of muscle activation, increasing the muscle force can be achieved two ways, either by increasing the maximum isometric force or increasing the level of activation. Therefore, the VMOS results suggest that increased neural VMO excitation may play an important role in reducing the lateral joint loading, although the ability of the nervous system to selectively activate muscles within a synergistic group is highly debated [6].

Previous work has identified a correlation between subjects with timing deficits between the VMO, VL and PFP [6,12], although the functional significance of the timing deficit (on the order of $5 \mathrm{~ms}$ ) has been questioned $[6,7]$. The results of the present study suggest that a $5 \mathrm{~ms}$ timing advance or delay is indeed important, although this theoretical result may depend on the time constants used in the muscle activation model. The timing advance reduced the peak and average force by $9.8 \%$ and $6.6 \%$, respectively. These reductions in the joint loading were similar in magnitude to the orthosis. An intriguing result was the effect of the timing delay. The peak and average lateral constraint force increased by $25.5 \%$ and $5.9 \%$, respectively. Although the timing delay does not have a great impact on the average force throughout the stance phase, it does significantly affect the peak force that corresponds with the impact peak (Fig. 3(b)). The impact peak force is suggested to be a leading cause of overuse injuries during repetitive movement tasks [40].

An interesting result was the relatively small and less consistent orthosis effect, especially for the peak lateral 
contact force. Proponents of orthoses have advocated orthoses as an important component in the treatment of PFP [17]. But the simulations showed that the orthosis examined in the present study was not as consistent as VMOS in reducing the joint loading. The lack of a stronger orthosis effect could be related to the specific orthosis used in the study, which did not change the shape of the foot-ground interface, but only increased stiffness in the arch area. This type of orthosis was chosen for this study because it is less likely to require adaptations in muscle activation patterns. Muscle coordination adaptation to the orthosis may be important and produce favorable or adverse effects. The advantage of the simulation model is that it allowed us to assess the effectiveness of the orthosis with all the parameters in the system preserved (e.g., muscle coordination). Further, the subjects used in the study were normal, healthy subjects without a history of PFP and most likely would not be candidates for the orthosis treatment. Specifically, the lack of orthosis effect in certain subjects may have been due to the fact that those subjects had no pressure under the medial arch during running. It is possible that a simulation control group of subjects with chronic PFP and candidates for orthoses would produce greater changes in the joint loading. The specific orthosis examined in the present study is also one of many types used to treat PFP. Future studies should be directed towards examining different configurations and their effectiveness towards reducing patellofemoral loads.

These findings showed that the orthosis treatment had a beneficial effect in some subjects and no effect in other subjects. An adverse effect was never observed. The effect of selective VMO strengthening was more consistent across subjects, but the feasibility of this treatment is debatable. Activation timing of the VMO muscle had a consistent effect on lateral patellofemoral loading, especially the peak force developed during the impact phase. The advantage of both the orthosis and strengthening treatments is that these do not require changing the complex neuromuscular coordination patterns of the patient. Future studies should be directed towards identifying techniques that selectively increase VMO strength as a potential treatment for PFP, and towards predicting the efficacy of orthotic treatment in patients based on their individual running mechanics.

\section{Acknowledgements}

This research was supported by NSERC of Canada, The Whitaker Foundation and Adidas America. We are thankful to Trisha Bellchamber and Phuong Ngo for collecting the experimental data.

\section{References}

[1] Matheson GO, Macintyre JG, Taunton JE, Clement DB, Lloyd-Smith R. Musculoskeletal injuries associated with physical activity in older adults. Med Sci Sports Exerc 1989;21:37985.

[2] van Mechelen W. Running injuries. A review of the epidemiological literature. Sports Med 1992;14:320-35.

[3] Clement DB, Taunton JE, Smart GW, McNicol KL. A survey of overuse running injuries. Phys Sports Med 1981;9:47-58.

[4] Cutbill JW, Ladly KO, Bray RC, Thorne P, Verhoef M. Anterior knee pain: a review. Clin J Sports Med 1997;7:40-5.

[5] Callaghan MJ, Baltzopoulos V. Anterior knee pain: the need for objective measurement. Clin Biomech 1992;7:67-74.

[6] Grabiner MD, Koh TJ, Draganich LF. Neuromechanics of the patellofemoral joint. Med Sci Sports Exerc 1994;26:10-21.

[7] Powers CM, Landel R, Perry J. Timing and intensity of vastus muscle activity during functional activities in subjects with and without patellofemoral pain. Phys Ther 1996;76:946-55.

[8] Lieb FJ, Perry J. Quadriceps function. An anatomical and mechanical study using amputated limbs. J Bone Joint Surg Am 1968;50:1535-48.

[9] Garrick JG. Anterior knee pain (chondromalacia patella). Phys Sports Med 1989;17:75-89.

[10] Mirzabeigi E, Jordan C, Gronley JK, Rockowitz NL, Perry J. Isolation of the vastus medialis oblique muscle during exercise. Am J Sports Med 1999;27:50-3.

[11] Werner S, Arvidsson H, Arvidsson I, Eriksson E. Electrical stimulation of vastus medialis and stretching of lateral thigh muscles in patients with patello-femoral symptoms. Knee Surg Sports Traumatol Arthrosc 1993;1:85-92.

[12] Voight ML, Wieder DL. Comparative reflex response times of vastus medialis obliquus and vastus lateralis in normal subjects and subjects with extensor mechanism dysfunction. An electromyographic study. Am J Sports Med 1991;19:131-7.

[13] Mariani PP, Caruso I. An electromyographic investigation of subluxation of the patella. J Bone Joint Surg Br 1979;61-B:169_ 71.

[14] Karst GM, Willett GM. Onset timing of electromyographic activity in the vastus medialis oblique and vastus lateralis muscles in subjects with and without patellofemoral pain syndrome. Phys Ther 1995; 75:813-23.

[15] MacIntyre DL, Robertson DG. Quadriceps muscle activity in women runners with and without patellofemoral pain syndrome. Arch Phys Med Rehabil 1992;73:10-4.

[16] Souza DR, Gross MT. Comparison of vastus medialis obliquus: vastus lateralis muscle integrated electromyographic ratios between healthy subjects and patients with patellofemoral pain. Phys Ther 1991;71:310-16.

[17] Eng JJ, Pierrynowski MR. Evaluation of soft foot orthotics in the treatment of patellofemoral pain syndrome. Phys Ther 1993;73:62-8.

[18] Bahlsen A. The etiology of running injuries: a longitudinal, prospective study. Calgary, AB: University of Calgary, 1988.

[19] Nigg BM, Cole GK, Nachbauer W. Effects of arch height of the foot on angular motion of the lower extremities in running. $J$ Biomech 1993;26:909-16.

[20] Tiberio D. The effect of excessive subtalar joint pronation on patellofemoral mechanics: a theoretical model. J Orthop Sports Phy Ther 1987;9:160-5.

[21] Eng JJ, Pierrynowski MR. The effect of soft foot orthotics on three-dimensional lower-limb kinematics during walking and running. Phys Ther 1994;74:836-44.

[22] Zajac FE, Gordon ME. Determining muscle's force and action in multi-articular movement. Exerc Sport Sci Rev 1989;17:187230 . 
[23] Cole GK, Nigg BM, van den Bogert AJ, Gerritsen KG. Lower extremity joint loading during impact in running. Clin Biomech 1996;11:181-93.

[24] Gerritsen KG, van den Bogert AJ, Nigg BM. Direct dynamics simulation of the impact phase in heel-toe running. J Biomech 1995;28:661-8.

[25] Wright IC, Neptune RR, van den Bogert AJ, Nigg BM. Passive regulation of impact forces in heel-toe running. Clin Biomech 1998;13:521-31.

[26] Neptune RR, Wright IC, van den Bogert AJ. A method for numerical simulation of single limb ground contact events: application to heel-toe running. Comput Methods Biomech Biomed Eng 1999 (in press).

[27] Delp SL, Surgery simulation: a computer graphics system to analyze and design musculoskeletal reconstructions of the lower limb. Ph.D. Dissertation, Department of Mechanical Engineering. CA (Stanford): Stanford University, 1990.

[28] Chandler RF, Clauser CE, McConville JT, Reynolds HM, Young JW. Investigation of inertial properties of the human body. Wright Patterson Air Force Base, Ohio (AMRL-TR-75-137), 1975.

[29] Clauser CE, McConville JT, Young JW. Weight, volume and center of mass of segments of the human body. Wright Patterson Air Force Base, Ohio (AMRL-TR-69-70), 1969.

[30] Inman VT. The joints of the ankle. Baltimore: Williams \& Wilkins, 1976, p. 1-117.

[31] Javadpour SM, Finegan PJ, O'Brien M. The anatomy of the extensor mechanism and its clinical relevance. Clin J Sport Med 1991;1:229-35.
[32] Soest AJv, Bobbert MF. The contribution of muscle properties in the control of explosive movements. Biol Cybern 1993;69: 195-204.

[33] Goffe WL, Ferrier GD, Rogers J. Global optimization of statistical functions with simulated annealing. J Econometrics 1994;60:65-99.

[34] Neptune RR, Hull ML. Evaluation of performance criteria for simulation of submaximal steady-state cycling using a forward dynamic model. J Biomech Eng 1998;120:334 41.

[35] Soderkvist I, Wedin PA. Determining the movements of the skeleton using well-configured markers. J Biomech 1993;26: 1473-7.

[36] Grood ES, Suntay WJ. A joint coordinate system for the clinical description of three-dimensional motions: Application to the knee. J Biomech Eng 1983;105:136-44.

[37] Wise HH, Fiebert IM, Kates JL. EMG biofeedback as treatment for patellofemoral pain syndrome. J Orthop Sports Phys Ther 1984;6:95-103.

[38] LeVeau BF, Rogers C. Selective training of the vastus medialis muscle using EMG biofeedback. Phys Ther 1980;60:1410-5.

[39] King AC, Ahles TA, Martin JE, White R. EMG biofeedbackcontrolled exercise in chronic arthritic knee pain. Arch Phys Med Rehabil 1984;65:341-3.

[40] Radin EL, Yang KH, Riegger C, Kish VL, O’Connor JJ. Relationship between lower limb dynamics and knee joint pain. J Orthop Res 1991;9:398-405. 